\title{
Three-dimensional analysis of free-electron laser performance using brightness scaled variables
}

\author{
M. Gullans, ${ }^{*}$ G. Penn, ${ }^{\dagger}$ J. S. Wurtele, and M. Zolotorev \\ Lawrence Berkeley National Laboratory, Berkeley, California 94720, USA
}

(Received 28 January 2008; published 11 June 2008)

\begin{abstract}
A three-dimensional analysis of radiation generation in a free-electron laser (FEL) is performed in the small signal regime. The analysis includes beam conditioning, harmonic generation, flat beams, and a new scaling of the FEL equations using the six-dimensional beam brightness. The six-dimensional beam brightness is an invariant under Liouvillian flow; therefore, any nondissipative manipulation of the phase space, performed, for example, in order to optimize FEL performance, must conserve this brightness. This scaling is more natural than the commonly used scaling with the one-dimensional growth rate. The brightness-scaled equations allow for the succinct characterization of the optimal FEL performance under various additional constraints. The analysis allows for the simple evaluation of gain enhancement schemes based on beam phase space manipulations such as emittance exchange and conditioning. An example comparing the gain in the first and third harmonics of round or flat and conditioned or unconditioned beams is presented.
\end{abstract}

DOI: 10.1103/PhysRevSTAB.11.060701

PACS numbers: $41.60 . \mathrm{Cr}$

\section{INTRODUCTION}

The three-dimensional linear theory of the free-electron laser (FEL) has been studied by numerous authors, and has recently been the subject of a comprehensive review [1] of the literature. Early studies examined optical guiding [2,3], but did not include the important effects of betatron motion and energy spread, and were restricted to round beams. The formalism used here is based on the work of Xie and coworkers [4], who developed a variational formalism, based on the linearized Maxwell-Vlasov equations, for FEL gain analysis. The formalism requires integration over the unperturbed characteristic orbits which includes the full three-dimensional wiggler-averaged electron motion, and uses dimensionless variables scaled with the onedimensional FEL growth rate. The most common application of this approach is a numerical parameter [5] fit to the FEL gain in terms of normalized emittance and energy spread. In this paper, we use a similar approach, but normalize the equations to a gain length that arises naturally from the six-dimensional beam brightness. In our analysis, we simultaneously include harmonic generation [6], round or flat beams $[7,8]$, and conditioned beams $[9,10]$.

The design optimization of an FEL requires the consideration of many tradeoffs between parameters for the electron beam and the undulators. Innovative ideas have been considered to enhance gain, for example, harmonic cascade FELs [11], amplification of seed pulses from laserbased harmonic generation, beam bunching [12], and beam conditioning. To achieve very short wavelengths, it may be more practical to extract a harmonic of the resonant wavelength rather than increase the beam energy or shrink the undulator period to reduce the resonant wavelength itself.

\footnotetext{
*Currently at Harvard University.

†gepenn@lbl.gov
}

By introducing beam conditioning [9], a correlation between electron energy and transverse amplitude, the negative impact of large transverse emittance can be drastically reduced. Choices of electron beam parameters, such as current, energy spread, transverse emittances, and beta functions, can drastically affect performance. Phase space manipulations such as bunch compression, conditioning, and emittance exchange can enhance performance but cannot increase the six-dimensional beam brightness. Thus, scaling the FEL equations by the beam brightness yields a convenient framework for evaluating the efficacy of these manipulations.

In Sec. II, the methods and formalism are introduced and in Sec. III, the dispersion relation in an integral form is derived. In Sec. IV, one-dimensional limits for the FEL gain are derived, and in Sec. V the flat beam limit is defined and example solutions are presented for various FEL configurations.

\section{METHODS AND FORMALISM}

As a starting point, we begin with the linear integral Eq. (1), which describes the small signal evolution of laser propagation in a high-gain FEL with arbitrary odd harmonics, $h$, of the resonant wavelength of a planar undulator. The dispersion relation is derived in Ref. [13] from an initial value formulation of the Maxwell-Vlasov system for the radiation field and the electron beam starting in the small signal regime, in which the Vlasov equation is solved to first order by integrating over unperturbed orbits.

Thus, the starting point for our analysis is

$$
\begin{aligned}
\left(\frac{\nabla_{\perp}^{2}}{2 h k_{1}}+\frac{i q_{h}}{2 L}\right) A(\boldsymbol{x})-\frac{4 i k_{u}^{2} \rho_{1 \mathrm{D}}^{3}}{h k_{1}}\left(\frac{a_{h}}{a_{1}}\right)^{2} \int_{-\infty}^{\infty} \mathrm{d}^{2} \boldsymbol{p} \\
\times \int_{-\infty}^{\infty} \mathrm{d} \eta \mathcal{F}_{h}(\boldsymbol{x}, \boldsymbol{p}, \eta)=0,
\end{aligned}
$$


where $\mathcal{F}_{h}$ is an eigenfunction for the perturbed distribution function in the linear regime:

$$
\mathcal{F}_{h}=h k_{1} \frac{\partial f_{0}}{\partial \eta} \int_{-\infty}^{0} \mathrm{~d} s e^{-i \mu_{h} s} e^{i h \psi_{0}(s)} A\left[x_{\beta}(s)\right],
$$

which is taken to evolve with distance as $e^{-i \mu_{h} z} \mathcal{F}_{h}$. Here, $f_{0}$ is the unperturbed electron distribution function, $\boldsymbol{x}_{\beta}(z)$ is the unperturbed orbit of an electron with initial coordinates $\boldsymbol{x}, \boldsymbol{p}$. The dimensionless, complex-valued growth rate, $q_{h}$, of the radiation power is related to the eigenvalue $\mu_{h}$ by

$$
\mu_{h}=i q_{h} / 2 L+k_{u} \Delta \nu_{h} .
$$

Specific choices for the length scale $L$ which is used to define the dimensionless $q_{h}$ will be presented in Sec. III. The power gain length, $L_{G}$, can be expressed in terms of this scale length by

$$
q_{r} \equiv \operatorname{Re}\left(q_{h}\right)=L / L_{G}
$$

The detuning $\Delta \nu_{h}=\left(\omega-h \omega_{1}\right) / \omega_{1}$, where $\omega_{1}=c k_{1}, k_{1}$ is fixed by the resonance condition $k_{1}=2 \gamma_{0}^{2} k_{u} /\left(1+a_{u}^{2}\right)$, $k_{u}=2 \pi / \lambda_{u}$, and $a_{u}$ is the normalized undulator strength.

The effective coupling strength of the electron motion to the $h$ th harmonic is, for $h$ odd, given by the usual Bessel function factor

$$
a_{h}=a_{u}(-1)^{(h-1) / 2}\left[J_{(h-1) / 2}(h \xi)-J_{(h+1) / 2}(h \xi)\right],
$$

where $\xi=a_{u}^{2} / 2\left(1+a_{u}^{2}\right)$. A particle with zero transverse amplitude has an average angle of $a_{u} / \gamma_{0}$, where $m_{e} c^{2} \gamma_{0}$ is the electron energy, $m_{e}$ is the electron mass, $c$ is the speed of light, and $a_{u}=e B_{\mathrm{rms}} \lambda_{u} / 2 \pi m_{e} c$ is the normalized strength of the undulator; here, $B_{\mathrm{rms}}$ is the rms value of the undulator field on axis. The average beta functions are denoted by $\beta_{x, y}$ and the focusing strengths by $k_{\beta x, y}=$ $1 / \beta_{x, y} . A(x)$ is the scaled transverse profile of the slowly varying monochromatic laser field, $E_{h}$, such that

$$
E_{h}(\boldsymbol{x}, z, t)=-\frac{4 h k_{1} \gamma_{0} m_{e} c^{2}}{\sqrt{2} e a_{h}} A(\boldsymbol{x}) e^{q_{h} z / 2 L} e^{i(k z-\omega t)} .
$$

In writing Eq. (1), we used the $\rho_{1 \mathrm{D}}$ parameter [14],

$$
\rho_{1 \mathrm{D}}=\left(\frac{\pi a_{1}^{2} r_{e} n_{e}}{4 \gamma_{0}^{3} k_{u}^{2}}\right)^{1 / 3} \simeq\left(\frac{a_{1}^{2} I / I_{\mathrm{A}}}{8 \gamma_{0}^{3} k_{u}^{2} \sigma_{x} \sigma_{y}}\right)^{1 / 3},
$$

where $n_{e}$ is the peak electron density on axis and $r_{e}=$ $e^{2} / 4 \pi \epsilon_{0} m_{e} c^{2}$ is the classical electron radius; alternatively, this expression is given in terms of the instantaneous current, $I$, and the Alfvén current $I_{\mathrm{A}}=e c / r_{e} \simeq$ $17.045 \mathrm{kA}$. The definition of $\rho_{1 \mathrm{D}}$ in terms of current and the rms transverse beam sizes, $\sigma_{x}$ and $\sigma_{y}$, assumes a Gaussian transverse profile.

The unperturbed orbits for the longitudinal coordinate, $\psi=\left(k_{1}+k_{u}\right) z-\omega t$, and the transverse phase space coordinates, $\boldsymbol{x}$ and $\boldsymbol{p}$, in the integral over past orbits in Eq. (2) are given by

$$
\begin{gathered}
\psi_{0}(s)=\left[2 k_{u} \eta-k_{1}\left(J_{x} / \beta_{x}+J_{y} / \beta_{y}\right)\right] s, \\
x_{\beta}(s)=x \cos \left(k_{\beta x} s\right)+\frac{p_{x}}{k_{\beta x}} \sin \left(k_{\beta x} s\right), \\
y_{\beta}(s)=y \cos \left(k_{\beta y} s\right)+\frac{p_{y}}{k_{\beta y}} \sin \left(k_{\beta y} s\right) .
\end{gathered}
$$

The longitudinal momentum, $\eta=\left(\gamma-\gamma_{0}\right) / \gamma_{0}$, appears differently from the other phase space coordinates in Eq. (2) because $\mathrm{d} \eta / \mathrm{d} z$ is already first order in the field [1].

We assume the beam is initially unbunched and matched to the undulator channel so that $f_{0}$ takes the form

$$
f_{0}\left(\eta, \boldsymbol{J}_{\perp}\right)=f_{\perp}\left(\boldsymbol{J}_{\perp}\right) f_{\|}\left(\eta \mid \boldsymbol{J}_{\perp}\right),
$$

where the transverse action, assuming a constant beta function in both directions, is given by $J_{x}=\left(x^{2}+\right.$ $\left.p_{x}^{2} / k_{\beta x}^{2}\right) k_{\beta x} / 2$, and similarly for $J_{y}$. In this discussion we have not assumed a round beam, thus almost doubling the number of beam parameters but maintaining enough generality for us to include flat beams in our analysis.

As in previous three-dimensional, analytic treatments of high-gain FELs, we assume that the focusing system is characterized by a constant beta function in both directions. Fortuitously, this approximation is justified for x-ray FELs when the cell length of the quadrupole lattice used for external focusing is much shorter than the average beta function $[15,16]$. This analysis is for a planar undulator; the case of a helical undulator only requires an appropriate change in the definition of $a_{h}$.

\section{DERIVATION OF THE DISPERSION RELATION WITH AN ARBITRARY SPATIAL SCALE}

We now specify the unperturbed electron distribution functions

$$
\begin{gathered}
f_{\|}\left(\eta \mid J_{\perp}\right)=\frac{1}{\sqrt{2 \pi} \sigma_{\eta}} e^{-\left(\eta-\kappa_{x} J_{x}-\kappa_{y} J_{y}\right)^{2} / 2 \sigma_{\eta}^{2}}, \\
f_{\perp}\left(J_{\perp}\right)=\frac{\sigma_{x} \sigma_{y}}{2 \pi \varepsilon_{x} \varepsilon_{y}} e^{-\left(J_{x} / \varepsilon_{x}+J_{y} / \varepsilon_{y}\right)},
\end{gathered}
$$

so that $f_{0}\left(\eta, \boldsymbol{J}_{\perp}\right)=f_{\perp}\left(\boldsymbol{J}_{\perp}\right) f_{\|}\left(\eta \mid \boldsymbol{J}_{\perp}\right)$. Here $J_{x, y}$ are the transverse actions and $\kappa_{x, y}$ are arbitrary conditioning parameters. The average values of $J_{x, y}$ in a beam are equivalent to the geometric emittances, $\varepsilon_{x, y}$.

Before introducing the brightness scaling, we continue using our unspecified length scale, $L$, which will be used to reduce longitudinal length scales to dimensionless form. We define the scaled variables $\tau=s / 2 L, X=x / \sigma_{x}, X_{\beta}=$ $x_{\beta} / \sigma_{x}$, and $\bar{\kappa}_{x}=\kappa_{x} / \kappa_{x}^{*}$, where $\kappa_{x}^{*}=k_{1} / 2 k_{u} \beta_{x}$ is the ideal conditioning parameter (in terms of maximizing the growth rate) in the 1D, cold beam limit (see Sec. IV). The following derivation closely follows that of 
Refs. [5,7], where a similar analysis is performed for an unconditioned beam. First we insert the conditioned distribution into the dispersion relation to arrive at

$$
\begin{gathered}
\left(\frac{\ell_{d x}}{2 h} \frac{\partial^{2}}{\partial X^{2}}+\frac{\ell_{d y}}{2 h} \frac{\partial^{2}}{\partial Y^{2}}+i q_{h}\right) A(\boldsymbol{X}) \\
=\int_{-\infty}^{\infty} \mathrm{d}^{2} \boldsymbol{X}_{\boldsymbol{\beta}} \Pi\left(\boldsymbol{X}, \boldsymbol{X}_{\boldsymbol{\beta}}\right) A\left(\boldsymbol{X}_{\boldsymbol{\beta}}\right),
\end{gathered}
$$

where

$$
\begin{aligned}
\Pi\left(\boldsymbol{X}, \boldsymbol{X}_{\boldsymbol{\beta}}\right)= & \int_{-\infty}^{0} \frac{C(L) \tau \mathrm{d} \tau e^{\theta-\varphi_{x}-\varphi_{y}}}{2 \pi \sin \left(\sqrt{\ell_{d x} \ell_{\varepsilon x}} \tau\right) \sin \left(\sqrt{\ell_{d y} \ell_{\varepsilon y}}\right)}, \\
\theta= & \left(q_{h}-i \ell_{\omega}\right) \tau-\left(h \ell_{\gamma}\right)^{2} \tau^{2} / 2, \\
\varphi_{x}= & {\left[1+i h \ell_{\varepsilon x}\left(1-\bar{\kappa}_{x}\right) \tau\right] } \\
& \times \frac{X^{2}+X_{\beta}^{2}-2 X X_{\beta} \cos \left(\sqrt{\ell_{d x} \ell_{\varepsilon x}} \tau\right)}{2 \sin ^{2}\left(\sqrt{\ell_{d x} \ell_{\varepsilon x}} \tau\right)},
\end{aligned}
$$

and where we have introduced the scaled parameters:

$$
\begin{array}{cc}
\ell_{d x}=\frac{2 L}{k_{1} \sigma_{x}^{2}} & \ell_{\varepsilon x}=2 L k_{1} \frac{\varepsilon_{x}}{\beta_{x}} \\
\ell_{\gamma}=4 L k_{u} \sigma_{\eta} & \ell_{\omega}=2 L k_{u} \Delta \nu_{h} .
\end{array}
$$

All of these definitions are identical for the $y$ direction with $x$ replaced by $y$. These parameters are scaled by $2 L$ just as we scaled the original integration variable, $s$, in the integral over unperturbed orbits, yielding a dimensionless growth rate $q_{r}=2 L \operatorname{Im} \mu_{h}$ [see Eq. (3)].

The prefactor, $C(L)$, in Eq. (13), also explicitly depends on the choice of scale length, and can be expressed as

$$
C(L)=2 L \frac{a_{h}^{2}}{2 h k_{1}} \frac{I / I_{\mathrm{A}}}{\varepsilon_{x}^{n} \varepsilon_{y}^{n} \sigma_{\gamma}} h^{2} \ell_{\gamma} \sqrt{\ell_{\varepsilon x} \ell_{\varepsilon y}},
$$

where the normalized emittances are $\varepsilon_{x, y}^{n}=\gamma_{0} \varepsilon_{x, y}$, and similarly we can define $\varepsilon_{z}^{n}=\sigma_{\gamma} \sigma_{z}$, where $\sigma_{\gamma}=\gamma_{0} \sigma_{\eta}$ and $\sigma_{z}$ is the bunch length, which is assumed to be much longer than a cooperation length. The fraction in $C(L)$ which depends on the current is proportional to the peak phase space density in that slice of the beam, and is related to the $6 \mathrm{D}$ beam brightness which may be defined as

$$
B_{N} \equiv \frac{8 \gamma_{0} k_{u}^{2} \rho_{1 \mathrm{D}}^{3}}{r_{e} a_{1}^{2}} \frac{\gamma_{0}}{\sigma_{\gamma}} \sqrt{\frac{\beta_{x}}{\varepsilon_{x}^{n}} \frac{\beta_{y}}{\varepsilon_{y}^{n}}}=\frac{I / e c}{\varepsilon_{x}^{n} \varepsilon_{y}^{n} \sigma_{\gamma}} \simeq \frac{N_{e}}{\varepsilon_{x}^{n} \varepsilon_{y}^{n} \varepsilon_{z}^{n}},
$$

where the last equality only applies for a flat current profile. The dimensions of $B_{N}$ are inverse volume, while scaling the current to the Alfvén current is equivalent to taking $r_{e} B_{N}$ which yields an inverse area.

To help understand Eq. (12), it is useful to note that

$$
\frac{J_{x}}{\varepsilon_{x}}=\frac{X^{2}+X_{\beta}^{2}-2 X X_{\beta} \cos \left(\sqrt{\ell_{d x} \ell_{\varepsilon x}} \tau\right)}{2 \sin ^{2}\left(\sqrt{\ell_{d x} \ell_{\varepsilon x}} \tau\right)} .
$$

The six (or, for a round beam, four) $L$-scaling parameters are the minimum number of parameters needed to scale the FEL equations; however, it is also possible to define

$$
\ell_{\beta x} \equiv 2 L / \beta_{x}=\sqrt{\ell_{d x} \ell_{\varepsilon x}}
$$

and, similarly, $\ell_{\beta y}$. Thus, there is ambiguity over which combinations of beam parameters to use in defining the dimensionless variables. We chose the $\ell_{\varepsilon}$ and $\ell_{d}$ parameters, instead of a combination involving the $\ell_{\beta}$ parameters, because the $\ell_{d}$ parameters are good measures of the strength of diffraction and are closely related to the transverse mode size, while the $\ell_{\varepsilon}$ parameters measure the angular spread of the beam similarly to how it appears [1] in the single-particle equation governing the ponderomotive phase, $\psi=\left(k_{1}+k_{u}\right) z-\omega_{1} t$,

$$
\mathrm{d} \psi / \mathrm{d} z=2 k_{u} \eta-k_{1}\left(J_{x} / \beta_{x}+J_{y} / \beta_{y}\right) .
$$

The remaining two parameters, $\ell_{\gamma}$ and $\ell_{\omega}$, are natural measures of the uncorrelated energy spread and the detuning, respectively.

Examining Eq. (14), we observe that the only remaining dependence on beam properties outside of the alreadydefined $L$-scaling parameters occurs within $C(L)$. The scale length $L$ must be chosen carefully in order to cancel out this dependence, but there are many ways to do this. We define the "brightness length" as

$$
\frac{1}{L_{B}} \equiv \frac{a_{h}^{2}}{2 h k_{1}} \frac{I / I_{\mathrm{A}}}{\varepsilon_{x}^{n} \varepsilon_{y}^{n} \sigma_{\gamma}}
$$

and for clarity denote the dimensionless scaled parameters which result from choosing $L=L_{B}$ as $b_{d, \varepsilon, \gamma, \omega}$. The prefactor then becomes

$$
C\left(L_{B}\right)=2 h b_{\gamma} \sqrt{h b_{\varepsilon x} h b_{\varepsilon y}} .
$$

One interesting feature of $L_{B}$ is that, being proportional to the beam brightness, most beam manipulations will either preserve this value or slightly lengthen it due to nonlinear effects, with the notable exception of beam cooling.

We remark that by using the $1 \mathrm{D}$ scaling length $L=$ $L_{1 \mathrm{D}} \equiv 1 / 2 \sqrt{3} k_{u} \rho_{1 \mathrm{D}}$, instead of $L=L_{B}$, we recover essentially the same scaling introduced in [5] with

$$
C\left(L_{1 \mathrm{D}}\right)=(2 / \sqrt{3})^{3} h\left(a_{h} / a_{1}\right)^{2} .
$$

When using this scaling, we will follow the notation of [5] and denote the scaling parameters as $\eta_{d, \varepsilon, \gamma, \omega}$. The only differences from our $L_{1 \mathrm{D}^{-}}$-scaling parameters and the corresponding parameters used in [5] is that, going from [5] to our scaling, $\eta_{d}$ becomes $4 \eta_{d}$ and $\eta_{\gamma}$ becomes $2 \eta_{\gamma}$. In general, the only manifestations of the different choices of $L$ appear in the prefactor, $C(L)$. This remains true in the various limits we obtain of Eq. (12) as well. Note that both choices of scaling parameter reduce the dispersion relation to functions of the previously defined scaling parameters, plus some dependences on the harmonic number which can 
also be scaled away if preferred. The major difference is that the prefactor is constant for the $L_{1 \mathrm{D}}$-scaling case while $L_{1 \mathrm{D}}$ itself is sensitive to the amount of beam compression, whereas in the $L_{B}$-scaling case the prefactor includes a functional dependence and $L_{B}$ is independent of the beam compression.

It remains to deal with the transverse derivatives in Eq. (12) by introducing the Fourier transform pair

$$
\begin{gathered}
\hat{A}\left(\xi_{x}, \xi_{y}\right)=\frac{1}{\sqrt{2 \pi}} \int_{-\infty}^{\infty} \mathrm{d}^{2} \boldsymbol{X} A(\boldsymbol{X}) e^{-i \boldsymbol{X} \cdot \boldsymbol{\xi}}, \\
A(X, Y)=\frac{1}{\sqrt{2 \pi}} \int_{-\infty}^{\infty} \mathrm{d}^{2} \boldsymbol{\xi} \hat{A}(\boldsymbol{\xi}) e^{i \boldsymbol{\xi} \cdot \boldsymbol{X}}
\end{gathered}
$$

Equation (12) then turns into the integral equation

$$
A(\boldsymbol{\xi})=\int_{-\infty}^{\infty} \mathrm{d}^{2} \boldsymbol{\xi}^{\prime} T\left(\boldsymbol{\xi}, \boldsymbol{\xi}^{\prime}\right) A\left(\boldsymbol{\xi}^{\prime}\right)
$$

where we have dropped the hats and introduced the kernel

$$
\begin{aligned}
T\left(\boldsymbol{\xi}, \boldsymbol{\xi}^{\prime}\right)= & 2 h^{2} b_{\gamma} \sqrt{b_{\varepsilon x} b_{\varepsilon y}} \int_{-\infty}^{0} \frac{\tau \mathrm{d} \tau e^{\left(q_{h}-i b_{\omega}\right) \tau-\left(h b_{\gamma}\right)^{2} \tau^{2} / 2}}{i q_{h}-\frac{b_{d x}}{2 h} \xi_{x}^{2}-\frac{b_{d y}}{2 h} \xi_{y}^{2}} \\
& \times \prod_{\ell=x, y} \frac{\exp \left(-\frac{\xi_{\ell}^{2}+\xi_{\ell}^{\prime 2}-2 \xi_{\ell} \xi_{\ell}^{\prime} \cos \left(\sqrt{b_{d \ell} b_{\varepsilon}} \tau\right)}{2\left[1+i h b_{\varepsilon \ell}\left(1-\bar{\kappa}_{\ell}\right) \tau\right]}\right)}{\sqrt{2 \pi}\left[1+i h b_{\varepsilon \ell}\left(1-\bar{\kappa}_{\ell}\right) \tau\right]} .
\end{aligned}
$$

Note that Eq. (19) has been used and $L_{B}$ has been chosen as the scale length. This integral equation can be numerically solved for the growth rate, $q_{h}$, and the Fourier transformed transverse mode profile, $A(\boldsymbol{\xi})$, thereby giving an exact solution to the FEL initial value problem for the $h$ th harmonic in the linear regime, assuming the unperturbed electron distribution is given by Eqs. (10) and (11). This has been performed by Xie [5] for a round, unconditioned beam at the fundamental wavelength, where it is found that the transverse profile of the fundamental mode, i.e., the mode with the highest growth rate, often has a Gaussian form similar to that of the electron beam.

The details of solving Eq. (23) are given in Appendix A. One way to visualize the results is to fix the emittance and beam brightness and vary the beta function and energy spread, while continuously adjusting the detuning to its optimal value. Ideally, this situation would be experimentally accessible using a method such as enhanced selfamplified spontaneous emission (ESASE) [12]. In this case, we choose to replace $b_{d}$ with the parameter $b_{\beta}=$ $2 L_{B} / \beta$, noting that $b_{\varepsilon} / b_{d}=k_{1}^{2} \varepsilon^{2}$ is fixed and $b_{d}=$ $b_{\beta}^{2} / b_{\varepsilon}$. Here, we solve the full variational equations (see Appendix A), while in the appropriate limits one can also use the fitting formula for the gain length developed by Xie in [5]. The results are shown in Fig. 1. There is a global optimum because increasing the $\beta$ function at fixed emittance decreases the current density, while decreasing the $\beta$ function shrinks the spot size so that eventually angular

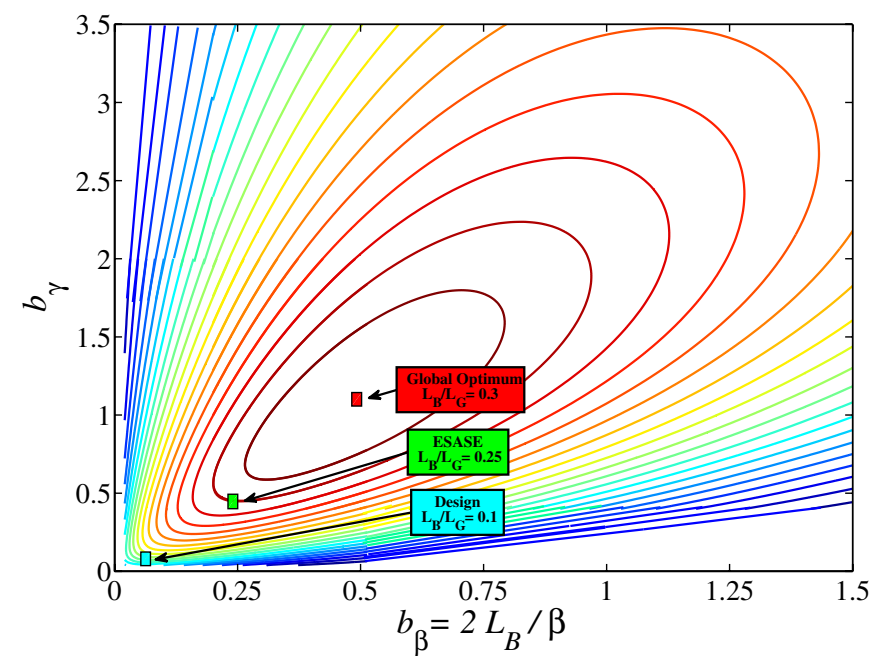

FIG. 1. (Color) Contour plot of dominant growth rate for fixed emittance and fixed beam brightness.

spreads in the beam and diffractive effects overwhelm the advantage of an increasing current density. Also shown are points corresponding to the Linac Coherent Light Source (LCLS) design, ESASE on the LCLS, and the optimal gain for a fixed emittance. The parameters for LCLS used here are a beam energy of $13.64 \mathrm{GeV}$, peak current of $3400 \mathrm{~A}$, normalized emittance of 1.2 micron, energy spread of $1.47 \mathrm{MeV}$. The undulator period is $3 \mathrm{~cm}$, the rms undulator parameter is 2.47 , the output wavelength is $0.15 \mathrm{~nm}$, and the corresponding brightness length $L_{B}=0.513 \mathrm{~m}$. The beta function is chosen to be $18 \mathrm{~m}$. The ESASE case has current and energy spread that are 10 times larger than the LCLS design, and the beta function is reduced to $4 \mathrm{~m}$. These beta functions give optimal results for the corresponding emittance, energy spread, and brightness. The LCLS FEL would have a shorter gain length if higher energy spread and smaller beta function were possible while preserving the nominal emittance at 1.2 micron, with the gain length improving from $4.5 \mathrm{~m}$ to an optimum of $1.8 \mathrm{~m}$. Note that because of the scaling parameter definitions, reducing the beta function also increases the parameter $b_{\varepsilon}$. In Sec. IV, we will show that if we consider substantially larger emittance and energy spread, the calculated gain length can improve even more and become close to the brightness scaling length, $L_{B}$. It is crucial to remember that this is at fixed brightness, so that the beam current is not fixed, and becomes extremely large at the optimum. In practice, this indicates that the limits on current set by the desire to preserve beam brightness during beam propagation and acceleration in the face of effects such as coherent synchrotron radiation (CSR) and wakefields are, in turn, limits on FEL performance. Furthermore, note that the benefits to LCLS of ESASE operation are only significant when $b_{\beta}$ (and thus $b_{\varepsilon}$ ) is increased to allow for tighter beam focusing. Otherwise, ESASE will increase the energy spread and not enhance 
the gain (i.e., move vertically in Fig. 1 to a less desirable operating point).

\section{ONE-DIMENSIONAL LIMITS}

The one-dimensional limit is found by first assuming symmetry in the two transverse planes $\left(b_{\varepsilon x}=b_{\varepsilon y}\right.$ and $\bar{\kappa}_{x}=\bar{\kappa}_{y}$ ), taking the transverse profile to be of the form $A(\boldsymbol{X})=A_{m}(R) e^{i m \varphi}$. By integrating over the polar angle, $\varphi$, one arrives at a brightness-scaled version of the integral equation found in Ref. [13], modified to include conditioning by the presence of the $(1-\bar{\kappa})$ factors as they appear in Eq. (23). Next, the desired limit is obtained by taking, at fixed brightness, both $b_{d}$ 's to zero in Eq. (23); physically, this corresponds to the limit of an infinite beam spot size with finite current density, and forces all modes to have a degenerate growth rate given by

$$
q_{h}+i 2 h^{2} b_{\gamma} b_{\varepsilon} \int_{-\infty}^{0} \frac{\tau \mathrm{d} \tau e^{\left(q_{h}-i b_{\omega}\right) \tau-\left(h b_{\gamma}\right)^{2} \tau^{2} / 2}}{1+i h b_{\varepsilon}(1-\bar{\kappa}) \tau}=0 .
$$

This limit becomes a good approximation when $b_{d} \ll q_{h}$ and $b_{\beta}=\sqrt{b_{d} b_{\varepsilon}}=2 L_{B} / \beta \ll 1$, such that the kernel of Eq. (23) becomes a convolution operator, i.e.

$$
T\left(\boldsymbol{\xi}, \boldsymbol{\xi}^{\prime}\right)=T\left(\boldsymbol{\xi}-\boldsymbol{\xi}^{\prime}\right),
$$

implying that we can inverse Fourier transform Eq. (23) and $A(\boldsymbol{X})$ becomes a multiplier on both sides of the equation.

The first condition, $b_{d} \ll\left|q_{h}\right|$, translates as $L_{G} \ll Z_{R}$, where $L_{G}$ is the power gain length and $Z_{R}$ is the Rayleigh range of a mode with a cross-sectional area equal to the beams, meaning that within a gain length most possible modes stay confined to the core of the beam. The second condition that $L_{G} \ll \beta$ means that the betatron oscillation is slow compared to a gain length so that the betatron mixing can be ignored. If both these conditions are met, the system becomes one-dimensional because it behaves like multiple local FELs that do not couple to each other. This is the reason for the degeneracy of the modes in this limit.

We begin by examining Eq. (24) in the case of a cold beam, where the energy spread and angular spread are sufficiently small that they have no impact on the gain length. Because the $6 \mathrm{D}$ phase space volume shrinks to zero in this limit, which at fixed brightness corresponds to zero particles, the brightness scaling is a less natural choice. Thus, for this limit only we shall employ the $L_{1 \mathrm{D}}$-scaling introduced in [5]. In this scaling, we use the four parameters $\eta_{d}=2 L_{1 \mathrm{D}} / k_{1} \sigma_{x}^{2}, \quad \eta_{\varepsilon}=2 L_{1 \mathrm{D}} k_{1} \varepsilon / \beta$, $\eta_{\gamma}=4 L_{1 \mathrm{D}} k_{u} \sigma_{\gamma} / \gamma_{0}$, and $\eta_{\omega}=2 L_{1 \mathrm{D}} k_{u} \Delta \nu_{h}$. The dispersion relation in the $1 \mathrm{D}$ limit takes the form

$$
q_{h}+i C\left(L_{1 \mathrm{D}}\right) \int_{-\infty}^{0} \frac{\tau \mathrm{d} \tau e^{\left(q_{h}-i \eta_{\omega}\right) \tau-\left(h \eta_{\gamma}\right)^{2} \tau^{2} / 2}}{1+i h \eta_{\varepsilon}(1-\bar{\kappa}) \tau}=0,
$$

where $C\left(L_{1 \mathrm{D}}\right)=(2 / \sqrt{3})^{3} h\left(a_{h} / a_{1}\right)^{2}$.
If $\eta_{\varepsilon}=0$ and $\eta_{\gamma}=0$, then we recover the well-known $1 \mathrm{D}$ cubic equation [14]

$$
q_{h}\left(q_{h}-i \eta_{\omega}\right)^{2}-i C\left(L_{1 \mathrm{D}}\right)=0 .
$$

An interesting feature of this equation is that it also applies in the case where $\eta_{\gamma}=0$ and $\bar{\kappa}=1$, i.e. $\kappa=k_{1} / 2 k_{u} \beta$, regardless of the value of $\eta_{\varepsilon}$. This coincidence is an expression of the physical fact that, with zero energy spread and in the absence of transverse variations in the radiation field, conditioning completely removes the longitudinal spread in ponderomotive phase that accumulates due to the varying betatron trajectories of the electrons. Furthermore, it can be shown that $\partial q_{r} /\left.\partial \bar{\kappa}\right|_{\left(\bar{\kappa}=1, \eta_{\omega}=0\right)}=0$ so that in this limit the natural conditioning parameter, $\kappa=$ $k_{1} / 2 k_{u} \beta$, maximizes the growth rate. In Appendix $\mathrm{B}$, the case of a conditioned beam with large emittance but zero energy spread is analyzed by going back to the variational equations while using the $L_{1 \mathrm{D}}$ scaling.

If we revert to scaling by $L_{B}$, the cold beam limit becomes

$$
q_{h}\left(q_{h}-i b_{\omega}\right)^{2}-i 2 h^{2} b_{\gamma} b_{\varepsilon}=0 .
$$

Thus, we find that the cold beam limit in this scaling requires linearizing the dispersion relation by keeping the factors $b_{\gamma}$ and $b_{\varepsilon}$ outside of the integral in Eq. (24), and approximating them as zero only within the integral. In this cold beam limit, the scaled growth rate will be proportional to $\left(b_{\gamma} b_{\varepsilon}\right)^{1 / 3}$ so long as diffraction remains unimportant. This scaling isolates the effect of the reduction in current that is associated with reducing the energy spread and emittance for fixed brightness.

We now consider warm beams, where $b_{\varepsilon}$ and $b_{\gamma}$ are large. For parameters such that the diffraction terms are still negligible, the growth rates for different transverse modes remain degenerate. In this regime, the main impact of large emittance on the dispersion relation is through introducing additional variations in the longitudinal velocity. We first rescale the integration variable in Eq. (24) to $z_{b} \equiv h b_{\varepsilon}^{c} \tau$ to obtain

$$
q+2 i \frac{b_{\gamma}}{b_{\varepsilon}^{c}} \int_{-\infty}^{0} \frac{z_{b} \mathrm{~d} z_{b} e^{\left(q / h b_{\varepsilon}^{c}-i b_{\omega} / h b_{\varepsilon}^{c}\right) z_{b}-\left(b_{\gamma} / b_{\varepsilon}^{c}\right)^{2} z_{b}^{2} / 2}}{(1-\bar{\kappa})\left(1+i z_{b}\right)}=0
$$

where $b_{\varepsilon}^{c} \equiv b_{\varepsilon}(1-\bar{\kappa})$ represents the effective angular spread of the beam. Now we define the ratios

$$
\begin{gathered}
\Gamma \equiv b_{\gamma} /\left|b_{\varepsilon}^{c}\right|=\frac{2 k_{u} \sigma_{\gamma}}{k_{1} \varepsilon^{n}|1-\bar{\kappa}| / \beta}, \\
\Omega \equiv-b_{\omega} / h b_{\varepsilon}^{c}=-\frac{k_{u} \Delta \omega_{h} / \omega_{1}}{h k_{1} \varepsilon(1-\bar{\kappa}) / \beta},
\end{gathered}
$$

and assume $|q| \ll h b_{\varepsilon}^{c}$, while keeping $\Gamma$ and $\Omega$ of order unity. This corresponds to a transversely warm beam, with at most imperfect conditioning. The result is a closed 
expression for the gain:

$$
q=2 i \Gamma \int_{0}^{\infty} \frac{z_{b} \mathrm{~d} z_{b} e^{-i \Omega z_{b}-\Gamma^{2} z_{b}^{2} / 2}}{|1-\bar{\kappa}|\left(1-i z_{b}\right)} .
$$

As the resulting $|q|$ has a global upper bound proportional to $1 /|1-\bar{\kappa}|$, the above approximation is consistent so long as $b_{\gamma}|1-\bar{\kappa}| \gg 1$. For an unconditioned beam, Eq. (32) is still a fair approximation for $b_{\gamma} \sim 1$. The failure of this limit to apply for ideal conditioning $(\bar{\kappa}=1)$ is related to the fact that the angular spreads which result from high emittance and small $\beta$ function do not significantly degrade FEL performance for a conditioned beam, and, as a result, the $\beta$ function is not subject to any lower bound. Note that this approximation cannot be extended in a consistent way to the case of a fixed, finite $b_{d}$, corresponding to fixing the spot size of the beam, but only works in the regime where diffraction is unimportant.

Now, by maximizing the real part of the right-hand sided of Eq. (32) with respect to $\Gamma$ and $\Omega$ we essentially find the maximum possible growth rate for a given brightness, undulator, and target wavelength. The results, including their scaling with the conditioning parameter, are

$$
\begin{aligned}
& q_{\mathrm{opt}} \approx(0.92+0.56 i) /|1-\bar{\kappa}|, \\
& \Gamma_{\mathrm{opt}} \approx 0.60, \quad \Omega_{\mathrm{opt}} \approx 1.3 .
\end{aligned}
$$

The power of these simple relations is illustrated by taking LCLS parameters for the energy spread and emittance. The value of $\Gamma_{\mathrm{opt}}$ implies that the beta function would be optimized at $\sim 25 \mathrm{~m}$, which is close to the design beta function.

Apart from the exceptional case of perfect conditioning, the conclusion that the growth rate is bounded is unsurprising; however, this analysis reveals that the primary condition for achieving a short gain length is high brightness rather than low emittance. If one can increase the emittance at fixed brightness (which requires increasing the current) while also optimizing the energy spread, then we see from Fig. 2 that the FEL performance actually improves to an asymptotic value, that is

$$
\lim _{b_{\varepsilon} \rightarrow \infty} q_{r}\left(b_{\varepsilon}, \Omega, \Gamma\right)>0,
$$

for some values of $\Gamma$ and $\Omega$. Furthermore, the maximum growth rate occurs in this asymptotic regime. This suggests that all the negative effects of a warm beam are captured by scaling the growth rate with $L_{B}$. Increasing the phase space volume at fixed brightness adds enough current to produce an overall positive effect (though with diminishing returns). In effect, the requirement for small emittances in practical systems derives from external constraints on the beam current rather than by the nature of the FEL interaction itself. The full dependence of the growth rate on $b_{\varepsilon}$ and $b_{\gamma}$ in the limit when diffraction is negligible is shown in Fig. 3, for fixed brightness and with no beam conditioning.

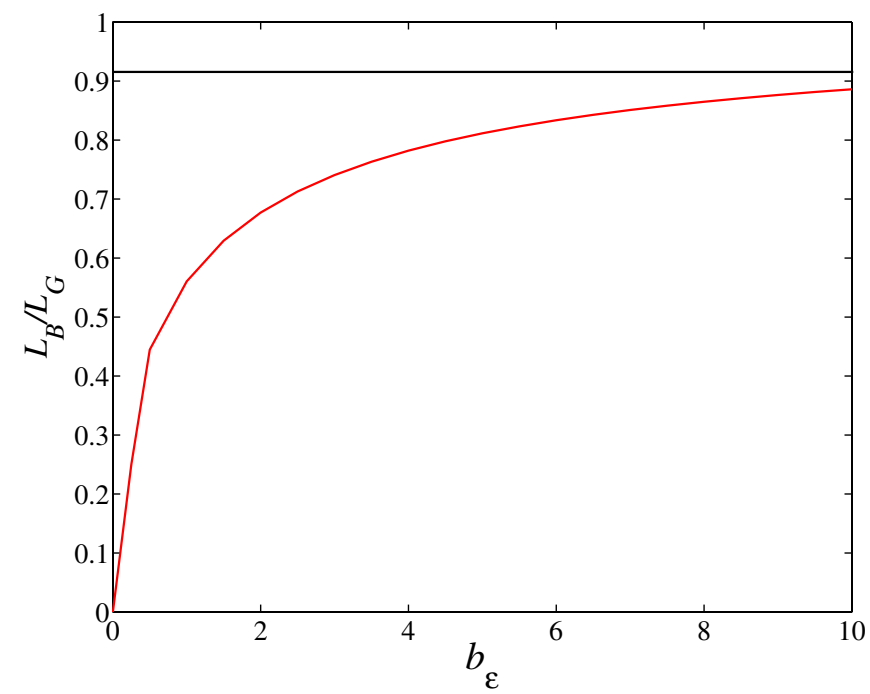

FIG. 2. (Color) Growth rate for fixed brightness as a function of emittance after optimizing over the energy spread. The effect of diffraction is assumed to be negligible.

We can make some sense of this asymptotic result by considering the phase equation, Eq. (17), and noting that increasing the beam phase space volume at fixed brightness amounts to stacking beams in phase space so that we continually populate the "conditioned" region of phase space where $\mathrm{d} \psi / \mathrm{d} z \approx 0$. The optimal value of $\Gamma$ then measures the relative strength of the two terms in Eq. (17) by specifying how phase space should be distributed to minimize the effect of dephasing across the beam.

The growth of the optimal detuning in proportion to the angular spread is expected because a more accurate resonance condition takes into account the effect of the betatron motion on the parallel velocity, so that

$$
\lambda=\frac{\lambda_{u}}{2}\left(\frac{1+a_{u}^{2}}{\gamma^{2}}+\frac{2 J_{x}}{\beta_{x}}+\frac{2 J_{y}}{\beta_{y}}\right)
$$

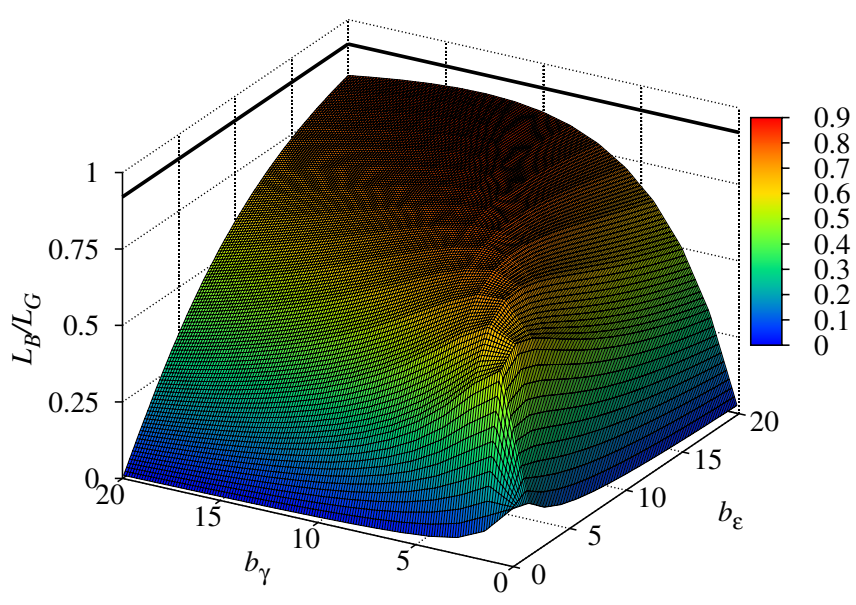

FIG. 3. (Color) Growth rate for fixed brightness as a function of energy spread and emittance parameters. The effect of diffraction is assumed to be negligible. 


$$
\langle\lambda\rangle=\frac{\lambda_{u}}{2}\left(\frac{1+a_{u}^{2}}{\gamma_{0}^{2}}+\frac{4 \varepsilon(1-\bar{\kappa})}{\beta}\right) .
$$

This further implies that the assumption $\left|\Delta \omega_{h} / \omega_{1}\right| \ll 1$ eventually breaks down; however, this should occur long after we are in the asymptotic region where we can ignore the $q$ dependence in the integral in Eq. (29).

Another observation is that the optimal detuning changes with conditioning parameter, to lower frequencies for $\bar{\kappa}<1$ and to higher frequencies for $\bar{\kappa}>1$. This can be seen from Eq. (36) and reflects the fact that the average longitudinal velocity normally decreases with increasing emittance, but for strong enough conditioning it increases.

We end our analysis of the one-dimensional limit by describing the behavior of the growth rate in various regimes. When both $b_{\varepsilon}|1-\bar{\kappa}| \ll 2$ and $b_{\gamma} \ll 1$, the growth rate is given by $L_{B} / L_{G}=\left[(3 \sqrt{3} / 4) h^{2} b_{\varepsilon} b_{\gamma}\right]^{1 / 3}$. For the case $h=1$, this is equivalent to $L_{G}=L_{1 \mathrm{D}}$, the standard cold beam limit, but expressed in terms of the brightness scaling parameters. For a fixed, small $b_{\varepsilon}<2 /|1-\bar{\kappa}|$, the growth rate scales like $b_{\gamma}^{1 / 3}$ for small $b_{\gamma}$, reaches a peak value at $b_{\gamma} \approx 1.2$ and then for large $b_{\gamma}$ scales as $1 / b_{\gamma}$. For a fixed, small $b_{\gamma}<1$, the growth rate scales like $b_{\varepsilon}^{1 / 3}$ for small $b_{\varepsilon}$, reaches a peak value at $b_{\varepsilon} \approx 2 /|1-\bar{\kappa}|$, and then decreases roughly inversely with $b_{\varepsilon}$. Note that the ratio of these two optimal values found by scanning in either parameter, $b_{\gamma} \approx 1.2$ and $b_{\varepsilon} \approx 2 /|1-\bar{\kappa}|$, defines the same ratio $\Gamma_{\text {opt }} \approx 0.6$ as found for the warm beam limit.

When both $b_{\varepsilon}$ and $b_{\gamma}$ are much larger than $1 /|1-\bar{\kappa}|$, the optimum growth rate is $L_{B} / L_{G} \simeq 0.92 /|1-\bar{\kappa}|$ and occurs whenever the ratio $\Gamma$ is approximately 0.6 . In the more general warm beam limit, the growth rate is a function of $\Gamma$ and $\bar{\kappa}$; when $\Gamma \gg 1$ and $b_{\gamma} \gg 1$, we find that $L_{B} / L_{G} \simeq \sqrt{2 \pi} e^{-1 / 2} /(\Gamma|1-\bar{\kappa}|) \simeq 1.52 b_{\varepsilon} / b_{\gamma}$, even for small $b_{\varepsilon}$. For small $\Gamma$, we estimate the growth rate as scaling roughly like $\Gamma /|1-\bar{\kappa}|$, although the warm beam approximation requires $b_{\gamma} \gg 1$, so the emittance parameter would have to be extremely large.

\section{ALTERNATIVE FEL OPTIONS}

We now consider various alternative FEL options besides using a conventional beam distribution operating at the fundamental resonant frequency. Specific examples are calculated to illustrate the contrast between conditioned and unconditioned beams, and to explore the possibilities for running an FEL at a higher harmonic of the resonant frequency. Before introducing these results, we first define the flat beam limit as an extreme contrast to the typical round beam profile used in FELs. Flat beams are an additional option which might be useful in managing wakefield effects or for use with small-gap undulators. A flat beam has sufficient asymmetry in the two transverse planes that it is possible for diffraction to be negligible in one plane but still significant in the other plane. This corresponds to the formal limit where $b_{d x}$ goes to zero while $b_{d y}$ remains nonzero and finite. A flat beam can be created via transverse emittance exchange, and in our example we take a fixed four-dimensional transverse phase space volume for the beam: $\varepsilon_{x} \varepsilon_{y}=$ (constant). In this case the brightness, $B_{N}$, remains constant, so that $L_{B}$ remains constant. The difficulty of achieving a focusing system that has vastly different beta functions in each direction and, simultaneously, keeps the beam stable, leads us to assume that $\beta_{x} \sim \beta_{y}$. Therefore, given that $b_{\beta}=\sqrt{b_{\varepsilon} b_{d}}=2 L_{B} / \beta$, we find that $b_{\beta y} \sim b_{\beta x}$, although $b_{d x} \ll b_{d y}$. This ordering only allows us to assert that the first condition for mode degeneracy in the $x$ direction is satisfied, and so the flat beam approximation also requires that we drop the betatron mixing effect in the $x$ direction. This is justified for two reasons, first because it has a negligible impact on the growth rate $(15 \%$ in the most extreme case for a round beam according to Appendix B) and second because $b_{\beta x} \ll b_{\varepsilon x}$ so that the effect is largely overwhelmed by the effect of angular spread in the beam (unless we condition in that plane).

In this approximation, the kernel of Eq. (23) takes the form

$$
T(\boldsymbol{\xi}, \boldsymbol{\xi})=T_{x}\left(\xi_{x}-\xi_{x}^{\prime}\right) T_{y}\left(\xi_{y}, \xi_{y}^{\prime}\right) .
$$

After assuming $A(\boldsymbol{\xi})=A_{x}\left(\xi_{x}\right) A_{y}\left(\xi_{y}\right)$, the integral over $\xi_{x}^{\prime}$ turns into a convolution. This implies that, just like the 1D limit, after Fourier transforming Eq. (23) back in the $x$ direction, $A_{x}(X)$ is a multiplier on both sides of the equation. Consequently, the $\xi_{x}$ dependence can be integrated out of $T$ to obtain the 1D integral equation

$$
A_{y}\left(\xi_{y}\right)=\int_{-\infty}^{\infty} \mathrm{d} \xi_{y}^{\prime} T_{F}\left(\xi_{y}, \xi_{y}^{\prime}\right) A_{y}\left(\xi_{y}^{\prime}\right),
$$

with the kernel

$$
\begin{aligned}
T_{F}= & \frac{2 h^{2} b_{\gamma} \sqrt{b_{\varepsilon x} b_{\varepsilon y}}}{i q_{h}-\frac{b_{d y}}{2 h} \xi_{y}^{2}} \int_{-\infty}^{0} \frac{\tau \mathrm{d} \tau e^{\left(q_{h}-i b_{\omega}\right) \tau-\left(h b_{\gamma}\right)^{2} \tau^{2} / 2}}{\sqrt{\left[1+i h b_{\varepsilon x}\left(1-\bar{\kappa}_{x}\right) \tau\right]}} \\
& \times \frac{\exp \left(-\frac{\xi_{y}^{2}+\xi_{y}^{\prime 2}-2 \xi_{y} \xi_{y}^{\prime} \cos \left(\sqrt{b_{d y} b_{\varepsilon y}} \tau\right)}{2\left[1+i h b_{\varepsilon y}\left(1-\bar{\kappa}_{y}\right) \tau\right.}\right)}{\sqrt{2 \pi}\left[1+i h b_{\varepsilon y}\left(1-\bar{\kappa}_{y}\right) \tau\right]} .
\end{aligned}
$$

A sampling of results for a soft $\mathrm{X}$-ray FEL is shown in Fig. 4, with all 3D effects included. The FEL design in these cases is for a fundamental wavelength of $3 \mathrm{~nm}$, an undulator period of $1 \mathrm{~cm}$, and an average beta function of $3 \mathrm{~m}$. The electron beam has a 0.5 micron normalized emittance, a 300 A peak current, and a $200 \mathrm{keV}$ energy spread. The flat beam examples have an emittance ratio of 100 and the same emittance product. The beam can be either flat or round, and conditioned or unconditioned, and the extracted wavelength can either be the $3 \mathrm{~nm}$ fundamental or the third harmonic at $1 \mathrm{~nm}$. Larger undulator parameters correspond to a larger resonant beam energy, and the range of $1<a_{u}<3$ shown in the figure corre- 


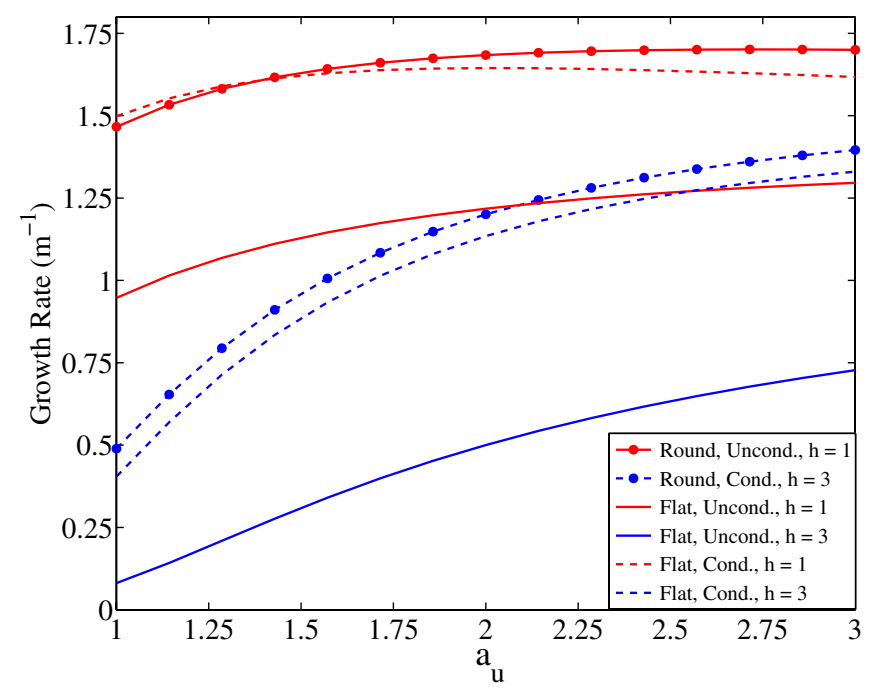

FIG. 4. (Color) Dependence of the growth rate on the undulator parameter $a_{u}$ (and hence beam energy) for various configurations of a soft x-ray FEL at $3 \mathrm{~nm}$ with $1 \mathrm{~cm}$ undulator period. Configurations vary between round or flat beams, beams with or without conditioning, and extraction of the fundamental or the third harmonic.

sponds to beam energies between 0.9 and $2.1 \mathrm{GeV}$. These examples serve to illustrate a few scaling properties of the gain length. Conditioned beams exhibit a small penalty in going from round to flat beams, while unconditioned beams have a larger discrepancy in performance. For higher harmonics to work near optimum requires $a_{u} \geq h$; for conditioned beams, different harmonics have similar gain lengths when $a_{u}$ is sufficiently large. For designs that are already optimized for unconditioned beams, the benefit of switching to conditioned beams is typically modest, with a reduction in gain length that is rarely greater than $25 \%$; however, even in these cases beam conditioning allows for relaxed values of $a_{u}$ and emittance, and is also favorable to the extraction of higher harmonics.

\section{CONCLUSION}

FEL performance has been analyzed in terms of new scaling parameters, where the gain length is measured against a length scale that is inversely proportional to the beam brightness. This gives a measure of the potential of an FEL which is independent of most electron beam manipulations except for beam cooling. We used this formalism to study conditioned beams, to compare round and flat beams, and to take various limits including cold beams, FELs where diffraction is negligible, and the 1D limit. The limit of large emittance and energy spread has also been studied, yielding an asymptotic solution which approaches the theoretical maximum growth rate as emittance is increased for fixed brightness. The optimal configuration is for a fixed ratio of the scaling parameters related to energy spread and emittance. Thus, the conventional wisdom that emittance limits FEL performance is not accurate, and in practice the inability to achieve brightness-limited performance is the result of other effects that constrain the peak electron current, such as wakefields and CSR.

We have also studied the effectiveness of ESASE or bunch compression, where the peak current is amplified in proportion to the energy spread. It is shown that the combination of transverse and longitudinal compression can significantly enhance the FEL gain, even when either technique alone does not improve performance. These beam manipulations are fairly straightforward, involving bunch stacking in phase space or bunch compression. We have shown that more complex techniques such as beam conditioning and emittance exchange can also greatly improve FEL performance. However, although such manipulations are Hamiltonian, technologies to implement them are unproven, and their costs and benefits should be compared with those of damping rings, which directly improve the beam brightness.

It is hoped that the methods discussed here will simplify the process of FEL optimization and will suggest new operating regimes. For example, beam conditioning is also shown to be useful in allowing for very small beta functions where the angular spread would otherwise reduce the growth rate. Thus, beam conditioning may be used to work around limitations in the gain length that result from constraints on the beam current. As beam brightness is a technologically limited input, the demonstration of a minimum gain length directly related to beam brightness should provide a useful yardstick for characterizing FEL performance.

\section{ACKNOWLEDGMENTS}

This work was supported by the Director, Office of Science, High Energy Physics, U.S. Department of Energy under Contract No. DE-AC02-05CH11231 and Grant No. DE-FG02-04ER41289.

\section{APPENDIX A: VARIATIONAL APPROXIMATIONS}

To actually solve the dispersion relation, Eq. (23), and calculate growth rates it is convenient to use the variational method developed by Xie [5,7]. We do this for an elliptical beam by assuming

$$
A(\boldsymbol{\xi}) \propto e^{-\xi_{x}^{2} / 4 \alpha_{x}-\xi_{y}^{2} / 4 \alpha_{y}},
$$

where $\alpha_{x, y}$ are variational parameters which are chosen to maximize $q_{r}$ (the real part of $q_{h}$ and thus the scaled growth rate). Some physical aspects of this mode which can be related to $\alpha$ are the Rayleigh range, $Z_{R}=$ $\left(L_{B} / b_{d}\right) \operatorname{Re}(1 / \alpha)$, and the radius of curvature of the phase fronts, $R=L_{B} /\left(b_{d} \operatorname{Im} \alpha\right)$.

After integrating both sides of Eq. (23) against $A(\boldsymbol{\xi})$ and applying the variational conditions $\delta q_{h} / \delta \alpha_{x}=0$ and $\delta q_{h} / \delta \alpha_{y}=0$, we arrive at the following set of equations: 


$$
\begin{aligned}
& F_{1}\left(q_{h}, \boldsymbol{\alpha}\right) \equiv \frac{i q_{h}}{4 \sqrt{\alpha_{x} \alpha_{y}}}-\frac{b_{d x}}{8 h} \sqrt{\frac{\alpha_{x}}{\alpha_{y}}}-\frac{b_{d y}}{8 h} \sqrt{\frac{\alpha_{y}}{\alpha_{x}}} \\
& -\int_{-\infty}^{0} \frac{2 h^{2} b_{\gamma} \sqrt{b_{\varepsilon x} b_{\varepsilon y}} \tau \mathrm{d} \tau e^{f_{1}}}{\sqrt{f_{2 x} f_{2 y}}}=0, \\
& F_{2}\left(q_{h}, \boldsymbol{\alpha}\right) \equiv \frac{\partial F_{1}}{\partial \alpha_{x}}=-\frac{i q_{h}}{8 \alpha_{x} \sqrt{\alpha_{x} \alpha_{y}}}-\frac{b_{d x}}{16 h \sqrt{\alpha_{x} \alpha_{y}}} \\
& +\frac{b_{d y}}{16 h \alpha_{x}} \sqrt{\frac{\alpha_{y}}{\alpha_{x}}}+\int_{-\infty}^{0} \frac{h^{2} b_{\gamma} \sqrt{b_{\varepsilon x} b_{\varepsilon y}} \tau \mathrm{d} \tau f_{3 x} e^{f_{1}}}{f_{2 x} \sqrt{f_{2 x} f_{2 y}}} \\
& =0 \text {, }
\end{aligned}
$$

and

$$
F_{3}\left(q_{h}, \boldsymbol{\alpha}\right) \equiv \frac{\partial F_{1}}{\partial \alpha_{y}}=0
$$

The function $F_{3}\left(q_{h}, \boldsymbol{\alpha}\right)$ is of the same form as $F_{2}$ with $x$ replaced by $y$. Apart from scaling parameters to the brightness length, $L_{B}$, and including conditioning and odd harmonics, these equations are of the same form as they appear in [7].

The same method can be applied to the equation obtained from the flat beam approximation, Eq. (37), in which case we find similar variational equations:

$$
\begin{aligned}
F_{1}\left(q_{h}, \alpha_{y}\right) \equiv & \frac{i q_{h}}{2 \sqrt{\alpha_{y}}}-\frac{b_{d y} \sqrt{\alpha_{y}}}{4 h} \\
& -\int_{-\infty}^{0} \frac{2 h^{2} b_{\gamma} \sqrt{b_{\varepsilon x} b_{\varepsilon y}} \tau \mathrm{d} \tau e^{f_{1}}}{\sqrt{f_{2 y}\left[1+i h b_{\varepsilon x}\left(1-\bar{\kappa}_{x}\right) \tau\right]}}=0,
\end{aligned}
$$

and

$$
\begin{aligned}
F_{2}\left(q_{h}, \alpha_{y}\right) \equiv & \frac{\partial F_{1}}{\partial \alpha_{y}}=-\frac{i q_{h}}{4 \alpha_{y}^{3 / 2}}-\frac{b_{d y}}{8 h \sqrt{\alpha_{y}}} \\
& +\int_{-\infty}^{0} \frac{h^{2} b_{\gamma} \sqrt{b_{\varepsilon x} b_{\varepsilon y}} \tau \mathrm{d} \tau f_{3 y} e^{f_{1}}}{f_{2 y}^{3 / 2} \sqrt{1+i h b_{\varepsilon x}\left(1-\bar{\kappa}_{x}\right) \tau}}=0
\end{aligned}
$$

In both cases,

$$
\begin{aligned}
f_{1}= & \left(q_{h}-i b_{\omega}\right) \tau-\left(h b_{\gamma}\right)^{2} \tau^{2} / 2 \\
f_{2 x}= & {\left[1+i h b_{\varepsilon x}\left(1-\bar{\kappa}_{x}\right) \tau\right]^{2}+4 \alpha\left[1+i h b_{\varepsilon x}\left(1-\bar{\kappa}_{x}\right) \tau\right] } \\
& +4 \alpha^{2} \sin ^{2}\left(\sqrt{b_{d x} b_{\varepsilon x}} \tau\right), \\
f_{3 x}= & 4\left[1+i h b_{\varepsilon x}\left(1-\bar{\kappa}_{x}\right) \tau\right]+8 \alpha_{x} \sin ^{2}\left(\sqrt{b_{d x} b_{\varepsilon x}} \tau\right),
\end{aligned}
$$

and the definitions are again the same for the $y$ direction with $x$ replaced by $y$. The round beam equations can be obtained from Eqs. (A2) and (A3) by removing the $x$ and $y$ dependence from the parameters.

\section{APPENDIX B: THREE-DIMENSIONAL EFFECTS IN FELS DRIVEN BY CONDITIONED BEAMS}

As electrons undergo betatron oscillations in the wiggler, this additional motion across the transverse radiation mode influences their interaction with the radiation field. This effect is included in the Maxwell-Vlasov analysis through the integral over past orbits [13] in Eq. (1); however, in previous treatments of beam conditioning it has been largely ignored. In this section we shall characterize the degradation in FEL gain caused by this effect by considering an "ultraconditioned" beam defined to be the limit, for a conditioned beam with $\kappa=\kappa^{*}$, as the emittance goes to infinity, while the beta function goes to zero, and the spot size, which scales as their product, remains constant. Note that optimal detuning stays bounded in this case despite arbitrarily large angular spreads, unlike the situation in Sec. IV, because the effect of conditioning is to put particles with large angles in resonance. For simplicity, we assume a round beam.

Although this ultraconditioned limit can be taken at any point in the analysis, it is convenient to do it after the variational approximation. In order to take the limit, we set the energy spread to zero and use the fact that the integral over past orbits becomes the derivative of a Laplace transform of a periodic function. In this case the first variational equation, the dispersion relation for a Gaussian mode, takes the form

$$
\frac{i q}{4 \alpha}-\frac{\eta_{d}}{4 h}-\frac{\mathrm{d}}{\mathrm{d} \bar{q}} \int_{0}^{\infty} \frac{C\left(L_{1 \mathrm{D}}\right) \mathrm{d} \tau e^{-\bar{q} \tau}}{1+4 \alpha+4 \alpha^{2} \sin ^{2}\left(\eta_{\beta} \tau\right)}=0,
$$

where $\bar{q} \equiv q-i \eta_{\omega}$ and $\eta_{\beta} \equiv \sqrt{\eta_{\varepsilon} \eta_{d}}=2 L_{1 \mathrm{D}} / \beta$. Here we use the $L_{1 \mathrm{D}}$-scaling because we have set the energy spread to zero and, as a result, the brightness scaling is less convenient. After several manipulations of Eq. (B1), including breaking the integral into periods of the sin function, taking the $\beta$ function to zero, and reevaluating the derivative, we arrive at the new set of variational equations:

$$
\begin{aligned}
& \frac{i q}{4 \alpha}-\frac{\eta_{d}}{4 h}+\frac{C\left(L_{1 \mathrm{D}}\right)}{2 \pi\left(q-i \eta_{\omega}\right)^{2}} \int_{0}^{2 \pi} \frac{\mathrm{d} \tau}{1+4 \alpha+4 \alpha^{2} \sin ^{2} \tau}=0 \\
& -\frac{i q}{4 \alpha^{2}}-\frac{C\left(L_{1 \mathrm{D}}\right)}{2 \pi\left(q-i \eta_{\omega}\right)^{2}} \int_{0}^{2 \pi} \frac{\left(4+8 \alpha \sin ^{2} \tau\right) \mathrm{d} \tau}{\left(1+4 \alpha+4 \alpha^{2} \sin ^{2} \tau\right)^{2}}=0 .
\end{aligned}
$$

These equations are valid when $\beta \ll L_{G}$, where $L_{G}$ is the gain length, and $\eta_{d} \ll \eta_{\varepsilon}$, which is why we call this the ultraconditioned limit. By solving Eqs. (B2) and (B3) in the case where diffraction is weak, i.e., small $\eta_{d}$, we find that the growth rate (at optimal detuning) is decreased by $\sim 15 \%$ compared to a zero emittance beam with $\eta_{\varepsilon}=0$. For diffraction-dominated or pencil beams, i.e., large $\eta_{d}$, the inclusion of this effect has a negligible impact on the growth rate. 
We remark that the inclusion of this betatron mixing effect in general results in an ideal conditioning parameter greater than the value $\kappa^{*}=k_{1} / 2 k_{u} \beta$ predicted from the $1 \mathrm{D}$, cold beam limit. However, it is a small correction so we shall not carry out a full analysis here.

Ultimately, this analysis characterizes the effects of this betatron mixing up to the order we have represented the betatron motion in the Vlasov equation. References [15,17] contain detailed discussions of how conditioning is affected by higher-order, oscillatory terms in the FEL equations arising from the use of strong focusing. In particular, they add the requirement to our analysis that $L_{C} \ll \beta$ in order to avoid deleterious effects from these higher-order terms, where $L_{C}$ is the cell length of the quadrupole lattice used for external focusing; thus, the ultraconditioned limit is only applicable when

$$
L_{C} \ll \beta \ll L_{G}
$$

However, as long as $L_{C} \ll \beta$, we have established an upper bound on the degradation $(15 \%)$ that this betatron mixing effect has on a conditioned beam as compared to a zero emittance beam.

[1] Z. Huang and K.-J. Kim, Phys. Rev. ST Accel. Beams 10, 034801 (2007), and references therein.

[2] E. T. Scharlemann, A. M. Sessler, and J. S. Wurtele, Phys. Rev. Lett. 54, 1925 (1985).
[3] K.-J. Kim, Phys. Rev. Lett. 57, 1871 (1986).

[4] Y.H. Chin, K.-J. Kim, and M. Xie, Phys. Rev. A 46, 6662 (1992).

[5] M. Xie, Nucl. Instrum. Methods Phys. Res., Sect. A 445, 59 (2000).

[6] R. Bonifacio, R. Corsini, and P. Pierini, Phys. Rev. A 45, 4091 (1992).

[7] M. Xie, Nucl. Instrum. Methods Phys. Res., Sect. A 507, 450 (2003).

[8] P. Emma, Z. Huang, K.-J. Kim, and P. Piot, Phys. Rev. ST Accel. Beams 9, 100702 (2006).

[9] A. M. Sessler, D. H. Whittum, and L-H. Yu, Phys. Rev. Lett. 68, 309 (1992).

[10] A. Wolski, G. Penn, A. Sessler, and J. Wurtele, Phys. Rev. ST Accel. Beams 7, 080701 (2004).

[11] J. N. Corlett, W. M. Fawley, G. Penn, A. A. Zholents, W. Wan, M. Reinsch, and J. S. Wurtele, in Proceedings of the 26th International Free Electron Laser Conference, 2004, Poster No. THPOS51, pp. 637-639.

[12] A. A Zholents, Phys. Rev. ST Accel. Beams 8, 040701 (2005).

[13] Z. Huang and K.-J. Kim, Phys. Rev. E 62, 7295 (2000).

[14] R. Bonifacio, C. Pellegrini, and L. Narducci, Opt. Commun. 50, 373 (1984).

[15] S. Reiche, Nucl. Instrum. Methods Phys. Res., Sect. A 445, 90 (2000).

[16] L. H. Yu, C. M. Hung, D. Li, and S. Krinsky, Phys. Rev. E 51, 813 (1995).

[17] Z. Huang, G. Stupakov, and S. Reiche, Report No. SLACPUB-11781, 2006. 